\title{
BRÉSILIENS AU LOUVRE : Observation des pratiques, Connaissance des publics et Réflexivité des Acteurs
}

\author{
Telma Lasmar Gonçalves ${ }^{1}$, Maria Gravari-Barbas ${ }^{1}$ and Nilson Alves de Moraes ${ }^{1}$ \\ ${ }^{1}$ Chefe do Departamento de Turismo e Hotelaria, Universidade Federal Fluminense, Brazil
}

\begin{abstract}
Dresser le profil des touristes est un outil fondamental en vue de proposer des orientations politiques, d'établir de nouveaux produits et services et de connaître les problèmes structurels et conjoncturels pouvant faire obstacle au bon déroulement de la visite et le niveau de satisfaction du visiteur. En prenant note de ce que le touriste pense, agrée ou désagrée, de sa provenance et de ses habitudes, il devient possible de réorienter des projets et de créer des services plus adaptés à la demande
\end{abstract}

Keywords: BRÉSILIENS AU LOUVRE, Observation

\section{Introduction}

Dresser le profil des touristes est un outil fondamental en vue de proposer des orientations politiques, d'établir de nouveaux produits et services et de connaître les problèmes structurels et conjoncturels pouvant faire obstacle au bon déroulement de la visite et le niveau de satisfaction du visiteur. En prenant note de ce que le touriste pense, agrée ou désagrée, de sa provenance et de ses habitudes, il devient possible de réorienter des projets et de créer des services plus adaptés à la demande.

Il en est de même en de ce qui concerne les musées: en connaissant, au moyen de l'analyse de ses réponses à une enquête, le profil du visiteur, ses représentations du musée et ses attentes, les institutions muséales peuvent offrir un service mieux adapté aux demandes des visiteurs.

D’après Tobelem (2013), les enquêtes auprès des publics des musées doivent procurer une évaluation de l'efficacité économique de l'institution mais surtout de son efficacité culturelle (scientifique, éducative, culturelle et sociale). Elles sont tout aussi bien un outil d'évaluation du marché, de connaissance des demandes des visiteurs, actuels et potentiels, d'aide à la prise de décisions et de support à la planification stratégique. Menées et analysées régulièrement, les enquêtes révèlent les tendances des pratiques de visite au cours du temps. Les résultats des enquêtes peuvent ainsi offrir des repères pour les programmes de fidélisation des visiteurs. Les enquêtes qualitatives peuvent permettre l'évaluation de programmes, de services, d'expositions, de la communication, de la perception des visiteurs tout autant qu'à mieux aménager aussi bien qu'un meilleur aménagement des espaces et l'amélioration de l'offre de services. «L'étude des visiteurs peux aussi avoir fonction de permettre de mieux décliner les moyens à mettre en œuvre pour optimiser l'offre muséale, le mettre en valeur, la présenter et la rendre attractive pour différents types de visiteurs, l'objectif n'étant pas nécessairement d'accroître la fréquentation» (TOBELEM, 2013, p. 235).

Les touristes des BRIC's (Brésil, Russie, Inde et Chine), attirent actuellement l'attention des chercheurs qui visent à connaître ces nouveaux clients et à savoir ce que représentent pour eux les grands « attracteurs culturels » tels que les grands musées français. Le Musée du Louvre, sujet de cette enquête, qui dispose du Baromètre des publics du Louvre vient de divulguer une recherche concernant le profil socio-économique de ses touristes. Cette enquête, barométrique, est de nature quantitative. Notre recherche, par le biais d'entrevues semi-structurées, vise 
à obtenir des connaissances plus qualitatives concernant la perception du musée par les touristes brésiliens, leurs représentations, et la façon dont ils ont «tiré profit» de cet équipement culturel.

Le nombre des Brésiliens voyageant à l'étranger a considérablement augmenté depuis le début du XXIème siècle, du fait principalement de la stabilité économique, de l'élargissement du crédit, de la réduction du coût du voyage et des taux de change favorables.

\begin{tabular}{|c|c|c|c|c|c|}
\hline & $\begin{array}{l}\text { DEMANDE } \\
\text { Monde } \\
\text { (millions) }\end{array}$ & (millions) & $\begin{array}{l}\text { A PARTICIPA } \\
\text { Brésil } \\
\text { (millions) }\end{array}$ & $\begin{array}{l}\text { Brésil en Améric } \\
\text { du Sud } \\
\text { (millions) }\end{array}$ & $\begin{array}{l}\text { S LE MONDE } \\
\text { Brésil dans le monde } \\
\text { (millions) }\end{array}$ \\
\hline 2005 & 803 & 18,3 & 5,4 & $29,3 \%$ & $0,7 \%$ \\
\hline 2006 & 847 & 18,7 & 5 & $26,7 \%$ & $0,6 \%$ \\
\hline 2007 & 904 & 20,2 & 5 & $25,0 \%$ & $0,6 \%$ \\
\hline 2008 & 922 & 20,8 & 5,1 & $24,3 \%$ & $0,5 \%$ \\
\hline 2009 & 882 & 22.9 & 4,8 & $23,4 \%$ & $0,5 \%$ \\
\hline 2010 & 940 & 24,4 & 5,2 & $22,0 \%$ & $0,5 \%$ \\
\hline 2011 & 982 & 25,8 & 5,4 & $20,9 \%$ & $0,5 \%$ \\
\hline 2012 & 1.035 & 26,9 & 5,7 & $20,0 \%$ & $0,5 \%$ \\
\hline 2013 & 1.087 & 27,4 & 6 & $22,0 \%$ & $0,5 \%$ \\
\hline 2014 & 1.138 & 29 & * & * & * \\
\hline
\end{tabular}

Source : élaboration des auteurs, d'après le site:

http://www.observatoriodoturismo.com.br/pdf/ANUARIO 2015 BASE 2014.pdf Accès: 15 jan. 2017

Selon les données révelées par le IBGE, le PIB brésilien de 2013 s'est accru de 2,3\% par rapport à l'année antérieure. D'après les actes de réunión du COPOM, l'économie brésilienne a atteint en 2013 une inflation de $5,91 \%$ (5,84\% en 2012). (Annuaire Statistique de l'a Infraero, 2013, p.6).

De plus en plus de Brésiliens qui, il y a quelques années, n'imaginaient même pas qu'il leur serait possible de connaître d'autres pays, entreprennent désormais des voyages internationaux. Les destinations favorites demeurent l'Argentine, la Disneyworld en Floride et le Caraibes. Mais des pays Européens tels que la France, l'Italie, le Royaume-Uni, le Portugal et l'Espagne sont fort recherchés. Des « nouvelles » destinations (Istanbul, Croatie, République Tchèque et Dubai) attirent de plus en plus de Brésiliens. La présence des Brésiliens est très visible dans les musées, les châteaux, les églises, les temples, les restaurants, les places, les plages, les rues, mélés aux touristes européens, américains et asiatiques.

Le nombre des touristes visitant la France est en augmentation : 60 millions en 1995; 82 millions en 2007; 85 millions en 2013 et l'on estime que ce chiffre atteindra 100 millions en 2020. D'après le Ministère de l'Environnement, de 1'Energie et de la Mer , 989.436 Brésiliens sont arrivés en France en 2014, bien derrière toutefois les États-Unis (6.609.269) et le Canada (1.920.808).

Ayant intérêt à connaître les touristes étrangers visitant Paris et leurs préférences, l'Office du Tourisme et des Congés de Paris mène régulièrement des recherches quantitatives. Selon la publication Chiffres Clés 2013, nous apprend que 90.300.000 passagers sont passés par les aéroports Paris-Charles de Gaulle et Paris-Orly; 29.300.000 personnes ont séjourné à Paris et les monuments et musées parisiens ont accueilli 72.100.000 visiteurs.

L'enquête de l'Office du Tourisme et de Congés de Paris, realisée auprès de 13.360 personnes, donne des éléments sur les pratiques des Brésiliens. Parmi les 468 Brésiliens interrogés, $73.7 \%$ déclarent préférer 
"découvrir la ville"; $67.3 \%$ "visiter des musées et des monuments" ; 13\% "faire des courses"; $11.8 \%$ connaître la "gastronomie"; et 14.1\% "visiter des parcs et jardins".

Eu égard au pourcentage élevé des Brésiliens voulant visiter des musées et des monuments et sachant que le Louvre compte parmi les musées les plus visités, nous avons posé comme but général de notre recherche la perception que les touristes brésiliens ont du Louvre.

La mise en place du Baromètre du Public du Louvre (enquête continue sur la perception et la satisfaction des visiteurs du Musée du Louvre) est fondée, selon CARVALHO (2005, p.26), sur l'hypothèse que "les études du profil du public sont essentielles pour tout autre savoir concernant le public". En effet, le musée se sert du résultat des enquêtes pour fonder les projets visant à améliorer le contact avec les visiteurs et les services qui leur sont offerts.

Dans le projet de modernisation du Musée du Louvre, nommé Le Grand Louvre (1989), la construction de la Pyramide visait à rendre plus aisé l'accès des visiteurs.Suite à l'analyse des recherches menées auparavant, les espaces d'exposition ont été élargis et les services d'accueil ont été considérés comme des espaces prioritaires. L'information, la réception et l'orientation des visiteurs (plans du Musée en langues différentes, dépliants des expositions, textes et légendes clairs, signalisation et autres ressources) furent l'objet de stratégies développées par des professionnels spécialisés en communication et design. Les activités culturelles complémentaires et celles des auditoires ont été revues, restructurées et diversifiées pour servir les différents niveaux de scolarité, professionnels et les personnes handicapées (CAILLET, 1996).

Dans une étude de mars 1995, Claude Fourteau écrit : «On peut considérer comme une donnée durable le doublement de la fréquentation qui s'est opéré au terme des six dernières années (de 2772948 visiteurs à 6165 118 visiteurs payants et gratuits). L'ampleur de la croissance se lit également sur une période plus longue : au cours des années 1990-1994, le musée a accueilli presqu'autant de visites (26 millions) que durant toute la décennie, déjà en pleine progression,des années 80 (29 millions). Autre indication significative : le musée du Louvre a reçu en 94 plus de visiteurs que la Tour Eiffel»(CAILLET, 1996: 138).

L'ouverture de la Pyramide a été structurée en vue d'un meilleur accueil au public. A cette occasion l'Association des Amis du Louvre a été développée; ses membres se sont vus offrir des avantages tarifaires et un accès plus facile, revendication ancienne des visiteurs exprimée dans les enquêtes. Suite aux résultats de l'enquête démontrant que les flux le plus importants des visiteurs avaient lieu pendant la matinée, le Musée du Louvre a établi une nouvelle politique tarifaire, réduisant de 50\% le prix d'accès après 15 heures (CAILLET, 1996 : 138).

Par ce qu'il représente historiquement et par le rôle qu'il joue actuellement, le Musée du Louvre est une institution de référence internationale. On sait qu'il est difficile d'établir des paramètres permettant de comparer l'origine des musées français et celle des autres musées dans le monde. Les musées français ont une spécificité d'origine car: [...] La Spécificité du musée français, par rapport aux autres pays européens, tient à ses origines. Sa construction repose sur la nationalisation des anciennes collections royales et sur les conquêtes militaires.[...] Car l'heritage français n'est plus, en 1793, soumis à l'autorité de la tradition des artistes, instruire le peuple et transmettre des leçons neuves à la posterité". (POULOT, 2008:7).

Cet esprit de "liberté révolutionnaire" est sans aucun doute ce qui fait que le Musée du Louvre, sous ses différentes gestions, se réinvente, se renouvelle, cherchant toujours à accomplir les idéaux d'accès démocratique et de qualité pour tous.

Notre recherche se situe à un double niveau: Il s'agit tout d'abord de saisir les attentes et les représentations des touristes brésiliens. Nous partons de l'hypothèse, qui reste à vérifier, qu'il y a des attentes et des pratiques qui caractérisent le public brésilien du fait de ses caractéristiques culturelles, linguistiques, des rapports historiques et actuels entre la France et le Brésil. 
Il s'agit à un deuxième niveau d'analyser la façon dont les acteurs du musée réagissent aux résultats de notre enquête: les prennent-ils en compte? Et si oui, de quelle façon? Jusqu'à quel niveau, et de quelle manière le musée du Louvre s'adapte-t-il à la demande de ce public spécifique?

Nous espérons certes que les réponses obtenues pourront servir à la Direction de la recherche et des collections et au Service études et recherches sur les publics du Musée du Louvre, de sorte qu'ils puissent poursuivre leur mission de bien accueillir les visiteurs. Le Louvre évolue désormais progressivement grâce à son adaptation graduelle au public mondial qui est le sien.

\section{Les enquêtes de public au Musée du Louvre}

En France, dans les années 1980, d'importantes recherches ont été menées dans quelques grands musées et centres d'expositions, tels que la Cité des Sciences et de l'Industrie, le Centre Georges Pompidou et le Palais de la Découverte. Les recherches se sont intensifiées pendant la décennie suivante, avec le programme REMUS financé par la Direction de Musées de France et par le Département de l'Information Scientifique et Tecnique. En 1990, la création de l'Observatoire Permanent des Publics - OPP, travaillant avec les grands musées nationaux à vocation touristique, tels que le Musée du Louvre, le Musée d'Orsay, le Musée Rodin et le Musée Picasso, tout autant qu'avec de petits musées d'art, d'histoire, d'histoire naturelle et technologiques, dans différentes régions de la France (SANTOS, 2000: p. 34) permet de systématiser ces études.

L'OPP était à même de suggérer des solutions pour parer à certains manques dans les musées en permettant, grâce à une recherche de longue durée, de porter au débat les opinions des visiteurs afin d'apporter des corrections aux pratiques muséographiques, communicationnelles et informationnelles, visant à améliorer la communication entres les visiteurs et le musée. La méthodologie de l'OPP consistait à analyser les variables les plus pertinantes du public: antécédents et caractéristiques de la visite (motivation, groupe, temps, etc.), aspects socio-démographiques et culturels, évaluation de la visite (satisfaction vis-à-vis des différents aspects), usage des services, envie de revenir etc."Enfin, l'analyse des réponses met en lumière des priorités, nécessaires pour établir une politique de public: savoir qui vient, et pourquoi, met en évidence l'absence de visiteurs potentiels. Plus globalement, le musée peut alors s'inscrire dans la politique culturelle de la ville, du Département ou de la Région, afin de participer, à part entière à leur développement culturel, mais aussi social et économique (LEHALLE, 1993, p. 12)

L’OPP a été créé dans le cadre par du Département des Musées de France afin d'éviter que seuls les renseignements quantitatifs soient pris en compte par les gérants des musées; il est devenu un outil de savoir qualitatif accessible au public, menant à l'évaluation du service public des pouvoirs locaux, à la création de la formation universitaire de médiation culturelle et au développement d'une réflexion concernant la médiation dans d'autres domaines artistiques et culturels des musées (CAILLET, 1996, p.139).

De 1994 à 2004, le Musée Du Louvre a travaillé en collaboration avec l'OPP.En 2005 le musée a créé un département spécifique de recherche sur les publics et leurs pratiques,le "Service d'études et recherche", en vue de lancer de façon régulière ses propres recherches, divisées en trois grandes lignes:

- études "stratégiques": des thèmes ponctuels, tels que les horaires de fonctionnement ou l'impact économique de la gratuité les dimanches;

- recherches barométriques: connaître le profil socio-économique du visiteur, individuel ou en groupe, ses impressions concernant le Musée et son offre éducative et culturelle;

- études de réception concernant les programmes éducatifs et culturels du Musée et les expositions temporaires .

Ainsi, "au sein de la Direction de la recherche et des collections du musée du Louvre, le service études et recherches sur les publics réalise des études et des recherches visant à approfondir la connaissance des publics, évaluer l'attraction et la réception de l'offre éducative et culturelle, et anticiper les tendances susceptibles d'influer sur la fréquentation du musée et ses usages . 
Nous avons analysé les recherches de profil de public menées par le Musée du Louvre au cours des dernières années, en vue de donner substance à notre propre recherche. Le Musée dispose d'indicateurs mensuels et annuels de toutes les expositions temporaires, des gratuités allouées, de la fréquentation de l'auditoire, des groupes scolaires, de l'origine des visiteurs français et étrangers, de la location des audio-guides, de la vente de publications, des groupes autonomes aux expositions permanentes, des groupes ayant des limitations de déplacement, des groupes aux conditions sociales défavorisées, du taux d'audience aux conférences, des valeurs de recette des entrées, des donnations, des locations d'espace, des ventes... Quoique plutôt quantitatives, ces données sont une source intéréssante pour l'analyse des résultats des comportements en rapport avec d'autres paramètres tels que la conjoncture économique. Des tableaux de fréquentation sont analysés régulièrement depuis 1992, en rapport avec des facteurs internes, tels que l'offre culturelle du Musée lui-même (par exemple, l'inauguration de nouvelles galeries), les évènements mondiaux (le 11 septembre 2001) et les circonstances nationales (comme les attaques terroristes à Paris en 1995 et 1996, la Coupe du Monde en 1998, pouvoir d'achat des ménages français, grèves, parmi d'autres) .

Le site institutionnel a été une de nos sources d'information concernant le profil et le nombre des visiteurs du Musée du Louvre. On y trouve les éléments obtenus dans la recherche Visite aux collections permanentes par régions et pays de résidence. Les estimations de fréquentation sont établies sur la base des enquêtes du Baromètre des Publics du Louvre, qui interroge tous les ans environ 10.000 visiteurs des collections permanentes âgés de plus de 11 ans. Les visiteurs en groupes scolaires tout autant que ceux qui profitent d'entrée gratuite lors d'événements exceptionnels (Nuit Blanche, Nuit des Musées, évènements de mécénat) ne sont pas interrogés. D'après la recherche publiée dans le Rapport d'Activité 2014, le Musée du Louvre a reçu cette annéelà 9.259.000 visiteurs: 8.845.000 aux collections permanentes; 290.000 aux expositions temporaires; 56.000 ont utilisé l'auditorium et 58.000 ont visité le Musée Eugène-Delacroix (rattaché au Louvre du point de vue administratif).

D'après la recherche de 2013, le public enquêté est constitué de 75\% d'étrangers (Etats-Unis 12\%; Chine 6\%; Brésil 5\%; Italie 4\%; Royaume-Uni 4\%; Allemagne 4\% Russie 4\%) et 25\% de Français. 55\% des répondants sont des femmes et $45 \%$ sont des hommes. La distribution des âges est assez équilibrée (moins de 18 ans:19\%; $31-45$ ans: 22\%; 18-25 ans: 21\%; 26-30 ans: 15\%; $46-59$ ans: $12 \%$; 60 ans ou plus: 11\%). Quant au profil, les résultats indiquent que $51 \%$ sont des employés, $19 \%$ des étudiants en général, $2 \%$ des chômeurs, $9 \%$ des retraités: $17 \%$ des étudiants universitaires et $2 \%$ exercent d'autres activités.

Enfin, le résultat des enquêtes témoigne d'un niveau élevé de satisfaction des visiteurs du musée du Louvre: satisfaction générale de la visite 97\%; qualité de l'accueil 96\%; confort 92\%; contenu perçu 94\%; signalisation $93 \%$.

Une autre donnée importante selon le BPL est celle de la fréquentation annuelle des touristes étrangers. En ce qui concerne les Brésiliens, l'analyse des résultats est intéressante:

\begin{tabular}{|l|l|l|l|l|l|l|l|}
\hline 2008 & 2009 & 2010 & 2011 & 2012 & $\mathbf{2 0 1 3}$ & $\begin{array}{l}\text { Variation } \\
\mathbf{2 0 1 3 / 2 0 1 2} \\
\text { en \% }\end{array}$ & $\begin{array}{l}\text { Variation } \\
\mathbf{2 0 1 3 / 2 0 1 2} \\
\text { en chiffres }\end{array}$ \\
\hline 328.684 & 270.289 & 411.586 & 367.080 & 370.006 & 313.070 & $-15 \%$ & -66.591 \\
\hline $\begin{array}{l}\text { Rétraction née de } \\
\text { la crise } \\
\text { économique } \\
\text { internationale }\end{array}$ & & & & $\begin{array}{l}\text { Début de la } \\
\text { crise } \\
\text { économique } \\
\text { au Brésil }\end{array}$ & & \\
\hline
\end{tabular}

Source : élaboration des auteurs, d'après les donnés du BPL 2013

Le nombre de Brésiliens visitant le Musée du Louvre décline au cours des dernières années du fait de la crise financière qui touche le Brésil depuis 2012, entraînant la réduction du pouvoir d'achat, et la hausse de 1'Euro par rapport au Real. Le Louvre a reçu 8.887.653 visiteurs en 2013 dont 3,6\% de Brésiliens, soit 313.070 touristes, moins 66.591 que l'année antérieure . 
La classification du "Top 10" des pays des touristes étrangers du Louvre montre à l'évidence ce déclin. L'approfondissement de la crise brésilienne nous incite à croire que le Brésil sera absent du TOP 10 des prochaines éditions.

\begin{tabular}{|l|l|l|l|}
\hline Classification & $\mathbf{2 0 1 1}$ & $\mathbf{2 0 1 2}$ & $\mathbf{2 0 1 3}$ \\
\hline $\mathbf{1}$ & Etats-Unis & Etats-Unis & Etats-Unis \\
\hline $\mathbf{2}$ & Brésil & Chine & Italie \\
\hline $\mathbf{3}$ & Australie & Brésil & Chine \\
\hline $\mathbf{4}$ & Italie & Italie & Allemagne \\
\hline $\mathbf{5}$ & Chine & Royaume-Uni & Royaume-Uni \\
\hline $\mathbf{6}$ & Espagne & Allemagne & Brésil \\
\hline $\mathbf{7}$ & Allemagne & Russie & Russie \\
\hline $\mathbf{8}$ & Royaume-Uni & Espagne & Australie \\
\hline $\mathbf{9}$ & Russie & Japon & Japon \\
\hline $\mathbf{1 0}$ & Japon & Australie & Benelux \\
\hline
\end{tabular}

Source : Élabotation de auteurs, d'après les données du BPL 2013

L'extension de la recherche permet aux gestionnaires du Louvre d'acquérir plus de connaissances sur le profil d'un nouveau et important touriste: les touristes en provenance des BRICs. Nous nous pencherons dans cette analyse sur les touristes brésiliens et comparaison avec ceux des BRICS. Les résultats que nous analyserons sont ceux de l'enquête de 2013.

\section{Quant à la façon de visiter}

3,0\% des Brésiliens font la visite du Musée en groupe d'excursion

$1,5 \%$ font la visite accompagnés d'un conférencier du Musée

$97,0 \%$ font la visite à leur compte

\section{Le jour où les Brésiliens viennent le plus} souvent

Le Mercredi - 23,2\%

Le Vendredi - 17,1\%

Le Dimanche - 16,4\%

Le Jeudi - $15,4 \%$

Le Lundi - $15,1 \%$

Le samedi - 12,2\%

\section{En ce qui concerne le niveau de} satisfaction

99\% des Brésiliens se déclarent satisfaits de la qualité du service d'accueil et d'information 93\% ont trouvé satisfaisantes les conditions de confort du Musée

\section{Quant à la façon d'acheter le billet}

$88 \%$ parmi les Brésiliens achètent leur ticket d'accès directement (au guichet ou par internet)

$11,8 \%$ ont accès gratuit car ils bénéficient de programmes de mécénat

$0,2 \%$ viennent quand leur billet a déjà été acheté par le guide ou l'agent du groupe

\section{Quant à la durée de visite des Brésiliens}

1 heure $-3,4 \%$

De 1 à 2 heures - 22,4\%

De 2 à 3 heures - 29,2\%

De 3 à 4 heures - 20,6\%

Plus de 4 heures, $24,4 \%$

* En moyenne, 3 heures et 8 minutes

95\% ont affirmé être satisfaits de l'apport culturel reçu pendant la visite

$89 \%$ ont approuvé les indications inscrites au plan du Musée et la signalisation.

Source : Élabotaion de auteurs, d'après les données du BPL 2013 


\section{Touristes internationaux brésiliens: qui sont-ils?}

Plusieurs auteurs qui ont fait des recherches et débattu le rapport entre le visiteur ou public et les musées, tels Bourdieu (2000), Canclini (1998) et Carvalho (2006), soutiennent la thèse selon laquelle la visite des musées n'est pas une habitude spontanée mais bien culturelle, qui se constitue par l'influence de la famille, de l'école et de l'environnement social. De même soutiennent-ils que l'intérêt des gens pour les musées s'accroit avec l'élévation du niveau scolaire, du niveau socio- économique et des connaissances historiques et artistiques. (Carvalho, 2005, p. 21)

Aussi peut-on dire que visiter les musées est une habitude culturelle et sociale bâtie sur l'expérience vécue et sur le savoir: "Visiter des musées est une action où trois contextes se croisent: le personnel, le social et le physique. Le contexte personnel comprend les intérêts, les motivations et la curiosité du visiteur, pouvant influencer décisivement les résultats de la visite. Le contexte social inclut les personnes qui font la visite ensemble, les contacts avec d'autres visiteurs et avec les fonctionnaires eux-mêmes, qui jouent un rôle important dans l'expérience qui est faite au musée. Le contexte physique comprend les traits architectoniques tout autant que les objets exposés et instruments contenus dans le musée et exerçant une influence puissante sur la visite". (SANTOS, 2000, p. 69)

Nous nous posons ainsi la question-socle de ce travail: qui est le touriste brésilien qui visite le Musée du Louvre? Cette connaissance préalable est essentielle pour analyser les impressions des Brésiliens concernant le Musée le plus renommé et le plus visité au monde. Aussi, que fait le Musée du Louvre pour mieux accueillir les touristes brésiliens?

On peut trouver des données utiles pour la compréhension du comportement et des perspectives des touristes internationaux brésiliens dans la recherche Enquête auprès du consommateur, menée au mois de septembre 2014. .

Parmi les enquêtés, 31,6\% ont fait part de leur intention de voyager au cours des six mois suivants. Les destinations touristiques nationales recoltaient $76,4 \%$ des choix contre $20,4 \%$ pour les destinations touristiques internationales (3,2\% d'indécis). Quoique la fréquence des voyages internationaux ait considérablement augmenté pendant les 20 dernières années, la principale destination de voyage reste toujours le Brésil, surtout pour les Brésiliens dont le revenu ne dépasse 2.742 euros. Au-dessus de ce niveau de revenu, l'intention de voyager à l'étranger monte à 46,4\% et varie selon l'âge : moins de 35 ans - 34,3\%; entre 35 et 44 ans - 28,0\%; entre 45 et 60 ans - 30,7\%; plus de 60 ans - 35,8\%. Et aussi selon le niveau d'études: 13,5\% des licenciés veulent voyager à l'étranger, alors que le pourcentage s'élève à $41,3 \%$ chez ceux qui ont le master ou le doctorat.

Les dépenses faites dans les musées, les spectacles et autres activités culturelles ont légèrement augmenté en $2012(+2,2 \%)$. La fréquentation des principaux centres culturels français est en hausse et il faut relever le succès du Musée du Louvre-Lens, ouvert au public en décembre 2012, pendant sa première année. Avec plus de 860.000 visiteurs sur une année, ce musée-ci se trouve parmi les 20 sites culturels les plus visités en France.

\section{Les Bresiliens Au Musée Du Louvre}

Le "service d'études et recherche" du Louvre dispose d'un nombre de données quantitatives particulièrement important, résultat d'enquêtes menées systématiquement depuis plusieurs années

Il est devenu ainsi évident qu'il fallait développer une recherche qualitative et non pas quantitative. Cette décision a été confortée par plusieurs rencontres avec des acteurs du Louvre : Mme. Yannick Lintz, Directrice du Département des Arts de l'Islam, Mme. Anne Krebbs, Chef du Service études et recherches sur les publics étrangers et M. Serge Leduc, Directeur de l'accueil du public et de la surveillance.

La décision fût alors prise de réaliser, dans le hall de la Pyramide du Musée, à la sortie des galleries Sully, Denon et Richelieu, 30 entrevues enregistrées, aux questions ouvertes et fermées, avec des touristes brésiliens. 


\subsection{Méthodologie de la recherche}

L'enquête fût menée les 23 et 25 mars et le 1er, 2, 4 et 5 avril, ces trois derniers jours étant la Semaine Sainte .

L'enquêteur a pris place dans le hall principal, dans l'aire nommée «Zones non muséographiques: Pyramides», tout près de la sortie des visiteurs de la galerie Denon. Le choix de cet emplacement s'est fait suite à l'observation des Brésiliens qui sortaient par cette galerie, où se trouve Mona Lisa. L'enquêteur prospectait également les cafés afin de localiser des touristes brésiliens. S'il en identifiait il leur demandait s'ils accepteraient d'être interrogés. Initialement, l'identification des touristes brésiliens se faisait par le répérage de la couleur du plan du Musée qu'ils portaient - en l'occurrence verte pour le portugais. Ce n'était toutefois pas la meilleure stratégie, car les Portugais utilisaient le même plan et le plan des Italiens était également vert, à peine un peu plus clair. L'enquêteur abordait finalement les visiteurs après avoir identifié qu'ils parlaient le portugais du Brésil.

L'enquête comprenait deux parties: l'une avec des questions fermées, l'autre avec des questions ouvertes, enregistrées, puis retranscrites. L'enquêteur faisait en plus des annotations sur le formulaire même de l'enquête.

Trente (30) personnes furent interrogées, 13 hommes et 17 femmes. Nul n'était seul : tous les interviewés étaient en couple, ou avec des amis, ou en groupe. On demandait alors qui répondrait aux questions.

Résultats par lieu de résidence:

\begin{tabular}{|l|l|l|l|l|l|}
\hline VILLE & ETAT & NOMBRE & VILLE & ETAT & NOMBRE \\
\hline Belém & PA & 1 & $\begin{array}{l}\text { Campo Novo do } \\
\text { Parecis }\end{array}$ & MT & 1 \\
\hline Fortaleza & CE & 1 & Sorocaba & SP & 1 \\
\hline São Luís & MA & 1 & $\begin{array}{l}\text { São José dos } \\
\text { Campos }\end{array}$ & SP & 1 \\
\hline Gravatá & PB & 1 & Uberlândia & MG & 1 \\
\hline Aracaju & SE & 1 & Rio de Janeiro & RJ & 6 \\
\hline João Pessoa & PB & 1 & São Paulo & SP & 1 \\
\hline Rondonópolis & MT & 1 & BeloHorizonte & MG & 2 \\
\hline Brasília & DF & 3 & RibeirãoPreto & SP & 1 \\
\hline Jataí & GO & 1 & Araraquara & SP & 1 \\
\hline Goiânia & GO & 2 & Curitiba & PR & 2 \\
\hline
\end{tabular}

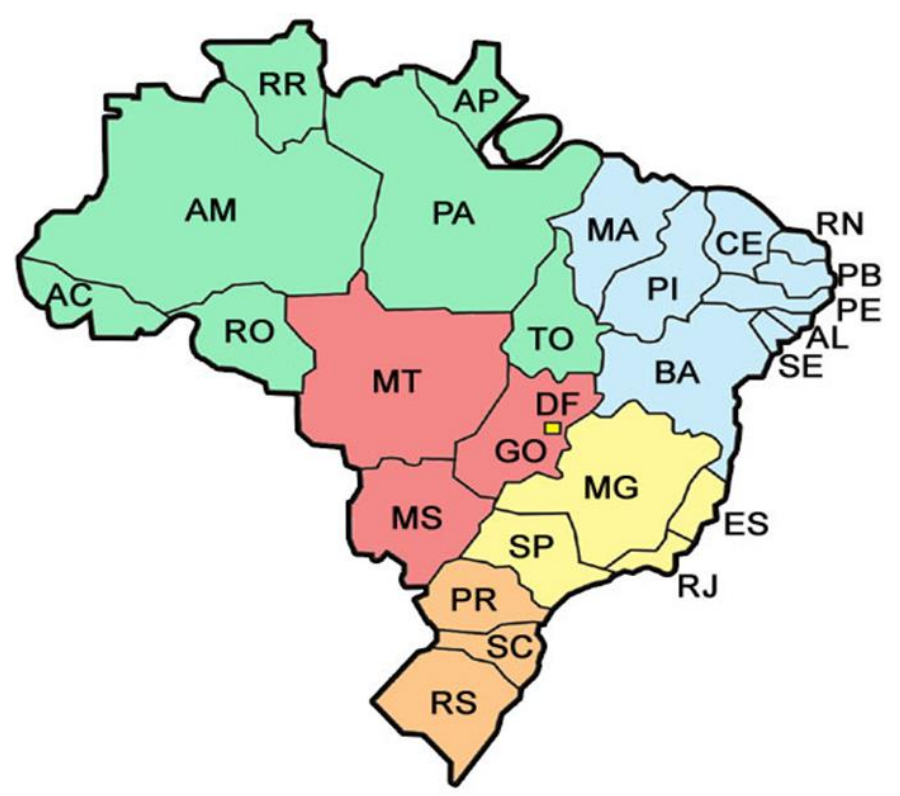


Les enquêtés venaient de 20 villes différentes, de toutes les régions du Brésil. Les villes au plus grande nombre de visiteurs furent Rio de Janeiro (6), Brasília (3), Belo Horizonte (2), Curitiba (2) et Goiânia (2).

Répartition par classe d'âge:

\begin{tabular}{|l|l|l|l|l|l|}
\hline 18 à 28 ans & 10 & 40 à 50 ans & 10 & 62 à 72 ans & 2 \\
\hline 29 à 39 ans & 6 & 51 à 61 ans & 2 & Plus de 73 ans & 0 \\
\hline
\end{tabular}

Répartition par niveau d'études:

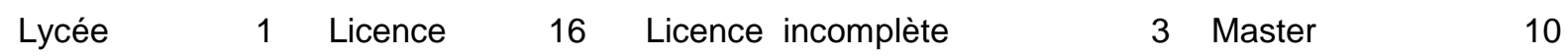

Les Brésiliens qui visitent le Musée du Louvre ont en général un haut niveau d'études. Parmi les 30 enquêtés, 16 disposaient d'un master et 10 d'un diplôme de Master. Ceux qui ont signalé une licence incomplète étaient des étudiants encore à l'université.

Enquêtés au sujet de leur venue à Paris, 17 ont répondu qu'ils y venaient pour la première fois et 13 étaient déjà venus avant (une fois, 3; deux fois, 5; trois fois, 3; 4 fois et plus, 2).

Eu égard à la durée de leur séjour en ville, voici les réponses : 3 jours, $2 ; 4$ jours, $10 ; 5$ jours, 7; 6 jours, $2 ; 7$ jours, $4 ; 8$ jours, $1 ; 9$ jours, 1; 14 jours, 1; 22 jours 2.

Concernant le logement en ville: 22 étaient logés à 1'hotel; 2 dans des auberges de jeunesse; 2 chez des amis ou familliers; 3 en appartement loué; 1 en aparthotel.

Enquêtés au sujet du type de voyage, la quasi-totalité (28 sur 30) a répondu que c'étaient leurs vacances. Deux enquêtés se trouvaient en voyage de noces.

Parmi les trente enquêtés, 10 sur 30 avaient acquis le Paris Museum Pass. Ceci n'impliquait pourtant pas forcément de visiter plus de musées, mais plutôt de visiter d'autres attractions culturelles, telles que l'Arc de Triomphe, la Sainte-Chapelle et le Panthéon. Concernant l'intention ou le fait de visiter d'autres musées pendant le voyage, voici les réponses:

\begin{tabular}{|l|l|l|l|l|l|}
\hline Musée d'Orsay & 17 & Musée de l'Orangerie & 5 & Musée Picasso & 4 \\
\hline Musée Marmottan Monet & 2 & Musée Guimet & 0 & Musée du Quai Branly & 0 \\
\hline Palais de Versailles & 16 & Beaubourg & 3 & Musée Rodin & 10 \\
\hline Fondation Louis Vuitton & 2 & Musée Carnavalet & 0 & & \\
\hline
\end{tabular}

Parmi les enquêtés certains ont affiché l'intention de visiter plus d'un musée; quatre ont répondu «aucun» à cette question. D'après l'analyse des questionnaires, ces quatre enquêtés ne séjourneraient que 4 jours à Paris, qu'ils visitaient pour la première fois. En ce sens, la visite du seul Musée du Louvre était presque obligée. A l'autre extrême, un interviewé a déclaré son désir de visiter le Palais Galliera, le Musée de l'Armée, le Musée de la Mode et des Tissus, le Musée des Arts Décoratifs, le Musée de la Marine, la Pinacothèque de Paris, le musée Jacquemart-André, la Cité de l'Architecture et du Patrimoine, le Palais de Fontainebleau et Les Invalides.

En ce qui concerne le moyen de transport utilisé pour arriver au Louvre, le métro vient en tête : 20 personnes contre 2 en bus, 2 en taxi, 5 à pied, 1 en «tuc tuc»

La deuxième partie de l'entrevue était composée de 2 questions fermées et de 10 questions ouvertes.

La recherche périodique menée par le Baromètre des Publics du Louvre - BPL montre que le temps moyen de visitation des Brésiliens au Musée du Louvre est 3 heures et 8 minutes. Voici notre résultat:

\begin{tabular}{|l|l|l|l|l|l|}
\hline Temps & Nombre & Temps & Nombre & Temps & Nombre \\
\hline $\begin{array}{l}\text { Entre 1 et } 2 \\
\text { heures }\end{array}$ & 6 & $\begin{array}{l}\text { Entre 2 et 3 } \\
\text { heures }\end{array}$ & 7 & $\begin{array}{l}\text { Entre 3 et 4 } \\
\text { heures }\end{array}$ & 8 \\
\hline $\begin{array}{l}\text { Entre 4 et } 5 \\
\text { heures }\end{array}$ & 6 & $\begin{array}{l}\text { Entre 5 et } 6 \\
\text { heures }\end{array}$ & 2 & Plus de 6 heures & 1 \\
\hline
\end{tabular}

Parmi les enquêtés qui sont restés le plus longtemps, deux répondirent qu'ils avaient fait une heure de queue dans l'accès à la Pyramide avant de pouvoir acheter leur ticket d'entrée. 
Concernant la façon dont le visiteur avait préparé la visite au Musée du Louvre (possibilité de réponses multiples), les voies les plus consultées furent les différents sites et le site du Musée du Louvre lui-même. Les blogs ont été plus consultés que les guides imprimés.

\begin{tabular}{|l|l|l|l|l|l|}
\hline $\begin{array}{l}\text { Consultation de } \\
\text { différents sites }\end{array}$ & 14 & $\begin{array}{l}\text { Consultation du site du } \\
\text { Musée du Louvre }\end{array}$ & 12 & $\begin{array}{l}\text { Consultation de guides } \\
\text { imprimés }\end{array}$ & 7 \\
\hline $\begin{array}{l}\text { Consultation } \\
\text { d'autres personnes }\end{array}$ & 6 & Consultation de blogs & 9 & Consultation de Plans & 1 \\
\hline $\begin{array}{l}\text { Aucune } \\
\text { consultation }\end{array}$ & 2 & $\begin{array}{l}\text { A assisté un documentaire } \\
\text { à la TV }\end{array}$ & 1 & Connaissait déjà le Louvre & 1 \\
\hline
\end{tabular}

Une question fondamentale pour la recherche concernait les motivations des visiteurs brésiliens (possibilité des réponses multiples).

\begin{tabular}{|l|l|l|l|}
\hline Connaître l'édifice & 24 & Connaître la collection & 19 \\
\hline Accompagner mon groupe & 2 & $\begin{array}{l}\text { Accomplir l'itinéraire de } \\
\text { l'excursion }\end{array}$ & 0 \\
\hline Voir une exposition temporaire & 0 & Autres & 15 \\
\hline
\end{tabular}

Dans la rubrique Autres, 10 personnes ont dit être venues pour voir Mona Lisa; 3 parce que le Louvre se trouve sur tous les itinéraires touristiques de Paris; 2 pour voir les œuvres principales; 1 pour connaître les Appartements de Napoléon III (croyant que c'était Napoléon Bonaparte!).

A la question «Quels obstacles avez-vous rencontrés dans votre visite au Musée?» 13 parmi les 30 enquêtés n'avaient rencontré aucune difficulté. Parmi ceux qui avaient rencontré quelque difficulté, les réponses se concentrent autour des faits qui suivent: queues trop longues; trop de monde; difficulté à l'entrée, pour trouver les WC, pour trouver les sorties et l'endroit où l'on vend Le Paris Museum Pass; signalisation en peu de langues; manque d'information concernant la gratuité pour les étudiants; petits caractères dans les légendes; accès difficile aux charriots et chaises roulantes; la taille du Musée. Une personne s'est plainte de l'audio-guide en espagnol trouvant l'appareil difficile à manier.

A la question «La circulation au Musée a été facile ou a-t-il été difficile de trouver les voies vers ce que vous aimeriez voir?», 16 personnes ont répondu qu'il est facile de suivre le plan et 4 ont dit que la signalisation du Musée est bonne.7 personnes, par contre, ont trouvé la circulation difficile et le plan (en papier) confus; le plan était trop petit et ne permettait pas d'établir le rapport entre le plan et le lieu. Une personne a dit avoir eu grand mal pour trouver la sortie des galeries. La réponse qui nous a le plus frappé a été: «je trouve que l'on devrait placer Mona Lisa plus près de l'entrée. Il faut marcher beaucoup avant d'arriver là où elle se trouve» (Licencée, femme, 18-28 ans).

Douze (12) personnes ont répondu «non» et 18 «oui» à question «avez-vous acheté quelque chose à manger au Musée»? Parmi ceux qui répondirent «oui», 6 enquêtés ont trouvé que c'était cher; 4 ont trouvé les prix honnêtes; 3 les ont trouvés en accord avec la ville; 5 les ont trouvés bons. Seule une personne a dit que les services étaient mauvais; toutes les autres les ont trouvés bons (10) ou très bons (7).

La question «Avez-vous utilisé un service du Musée - magasin, librairie, WC ou autre? Que pensez-vous de la qualité et des prix des services du Musée?»a reçu 8 «non» et 22 «oui», concernant surtout les WC. L'évaluation a été mauvaise, car seuls 9 enquêtés ont estimé qu'ils étaient propres. Les plaintes les plus fréquentes concernaient la saleté (7) et les queues (5). Un enquêté s'est plaint du manque d'eau pour se laver les mains, un autre de l'inadéquation des WC au nombre de leurs utilisateurs et deux ont trouvé que les WC étaient très malodorants. Il n'y a eu qu'un visiteur qui se soit rendu au magasin du Musée. Nous supposons que cela est dû au fait qu'au moment de l'enquête le magasin avait été déplacé vers l'extérieur du Musée du fait du chantier du Projet Pyramide 2014-1016. 4 personnes sont allées à la librairie et ont trouvé que les prix étaient bons et qu'elles avaient été bien reçues. Un enquêté a déclaré que «le service est attentif et efficace au vestiaire» (Licence, homme, 18-28 ans). Et un autre a dit que le garçon qui l'avait accueilli au guichet de Renseignement avait été grossier et lui avait parlé sans même le regarder (Licencée, femme, 62-72 ans). 
A la question «Qu'est-ce qui fût le plus marquant dans cette visite et restera enregistré dans votre mémoire?» les réponses ont été bien diversifiées, ce qui était attendu. Certaines réponses étaient prévisibles: l'intérieur et l'extérieur de l'édifice (9); Mona Lisa (5); les appartements de Napoléon III (5); la Pyramide (4); la peinture italienne (4); le nombre de gens et d'œuvres (3); le plafond de la galerie d'Apollon et les dimensions de l'édifice (2). Une réponse surprenante: «Je croyais venir voir des choses très anciennes; Je ne savais pas qu'il y avait cette partie nouvelle - (La Pyramide)» (Master, homme, 29-39 ans).

La diversité caractérise également les réponses à la question «Qu'est-ce que le Musée du Louvre signifie pour vous?».

Eu égard à la variété des réponses, le Musée du Louvre signifie en général Culture, Histoire de l'humanité, Beauté et la plus grande et la plus importante collection au monde. Quatre réponses sont plus personnelles : « Réalisation d'un vieux rêve» (Licencée, femme, 40-50 ans). «Une découverte. Chez nous il n'y a rien comme cela» (Master, homme, 29-39 ans). «C'est la principale référence dans la muséologie mondiale. Il possède l'une des collections les plus importantes au monde. C'est une référence de beauté, d'efficacité et d'évolution de la muséologie» (Licencée, femme, 62-72 ans). «Le musée est la grandeur et la puissance de la France. La France apprécie la culture et son patrimoine» (Master, homme, 40-50 ans).

Interrogés si «l'expérience de visiter le Louvre est différente de l'expérience de visiter des musées au Brésil ? $\mathrm{Si}$ oui, pourquoi?» tous ont répondu «oui». La taille imposante de l'édifice, la diversité et la qualité de la collection, le nombre de visiteurs et surtout l'organisation constituent les différences principales. Nous relevons cinq commentaires: «Au Brésil les musées sont très mauvais et n’ont presque rien» (Licencée, femme, 40-50); «C'est incomparable. Le niveau de la culture au Brésil est bien bas» (Licence, homme, 18-28 ans). «Au Brésil il n'y a rien de comparable: le Louvre est plein d'énergie, de touristes du monde entier, de joie» (Licencée, femme, 40-50 ans). «Le Louvre est unique, gigantesque, avec des œuvres importantes» (lycée, homme, 18-28 ans). «A cause de tout ce qu'il signifie, de l'envie d'y revenir et d'essayer de mettre en pratique ce qu'on a vu» (Licencée, femme, 62-72 ans).

A la question «Conseilleriez-vous la visite du Musée du Louvre à quelqu'un?» la presque totalité (29) a répondu «oui». L'un des enquêtés a répondu: «Non. Le Musée est très grand et on perd trop de temps. Cela dépend de celui qui vient et de ses intérêts» (Licence, homme, 18-28 ans).

Questionnés si «Lors d'un autre voyage à Paris retourneriez-vous au Musée du Louvre ?» 27 personnes ont répondu «oui»; 1 a répondu «peut-être» (Licencée, femme, 18-28 ans); 1 a répondu «Je ne sais pas, cela dépendra du temps disponible» (Lycée, femme, 18-28 ans); La même personne ayant répondu qu'elle ne conseillerait pas la visite au Musée a répondu à cette question par un «non» catégorique.

La dernière question était la suivante: «Avez-vous reçu quelque attention spéciale du fait d'être Brésilien? Avez-vous utilisé des moyens en langue portugaise aidant à la compréhension du Musée et des expositions?», 26 n'ont reçu aucun accueil différencié; 4 ont dit avoir été attentivement accueillis du fait d'être Brésiliens.Les enquêtés ont dit: «le fonctionnaire du guichet m'a demandé si je parlais anglais» (Licence, homme,18-28 ans); «les foncionnaires qui nous remettent l'audio-guide sont gentils et s'excusent du fait qu'il n'existe pas en portugais» (postuniversitaire, homme, 29-39 ans); «Le fonctionnaire des Reinseignements a demandé 'Tudo bem?'et il a remercié en portugais» (Lycée, femme, 18-28 ans); «Au bar et au vestiaire les fonctionnaires ont remercié en portugais» (BAC, femme, 40-50 ans). 28 personnes ont utilisé le plan en portugais et 2 n'ont pas utilisé de plan, 27 n'ont pas utilisé l'audio-guide et 2 l'ont utilisé en anglais et 1 en espagnol.

Pour finir, l'enquêté était prié de dire en trois mots ce qu'il pensait du Musée du Louvre. Les expressions ont été diversifiées mais peuvent en général se résumer comme suit: 


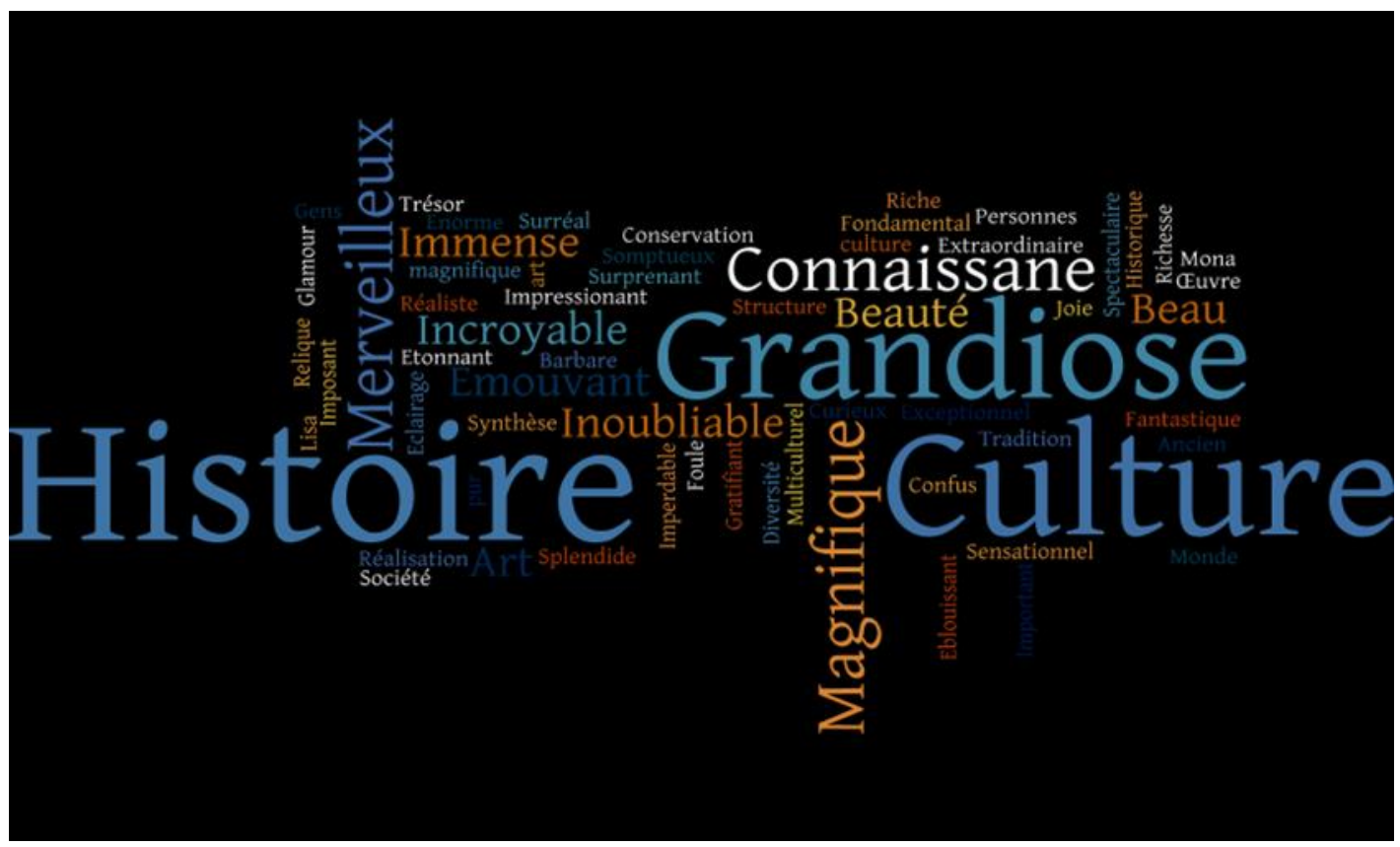

Le mot «Histoire» a été évoqué 8 fois; «Culture», 7 fois; «Grandiose», 6 fois; «Magnifique», «Merveilleux» et «Connaissance», 3 fois; «Incroyable», «Emouvant», «Impréssionant», Inoubliable», «Beau», «Immense», «Beauté», 2 fois.Les autres mots ne sont cités qu'une seule fois.

\section{Considérations Finales}

Les Brésilens qui voyagent à l'étranger sont des adultes, en vacances, jouissant d'un niveau d'études élevé et accompagnés de la famille ou des amis.

Les résultats de notre recherche concernant le profil du touriste brésilien sont compatibles avec ceux de l'enquête du Baromètre des Publics du Louvre- BPL: nous avons enquêté un nombre plus grand de femmes que d'hommes, la principale incidence du niveau d'âge est la même, seul un parmi les enquêtés se trouvait en groupe, quoique tous de la même famille; aucun enquêté n'était en excursion et le mercredi est le jour où le musée reçoit plus de Brésiliens.

La durée moyenne de visite des Brésiliens, d'après le BPL, est de 3 heures et 8 minutes. Parmi nos 30 enquêtés, celui qui est resté moins de temps - 1 heure - au Musée avait pour seul but voir Mona Lisa; ceux qui sont restés plus longtemps - 5 heures et 50 minutes et 6 heures et trente minutes - ont dit avoir attendu 1 heure à la queue et avoir interrompu la visite pour manger.

Tous les mots qualifiant le Musée du Louvre ont été positifs. Même les plaintes par rapport au plan confus, à la foule, aux WC sales ne compromettent pas l'impression finale concernant le Musée: il est en effet incontournable et une expérience frappante pour les Brésiliens, qui conseillent la visite et y retourneraient. L'offre de l'audio-guide en langue portugaise rendrait la visite au Musée du Louvre encore plus gratifiante et complète.

Pour tout ce qu'il représente, le musée du Louvre est une destination incontournable pour les touristes du monde entier qui visitent Paris.Il ne pourrait pas être différent pour les touristes brésiliens: il n'y aaucune chance d'aller à Paris sans aller au Louvre! Le musée dispose de quatre icônes impérieuses: Mona Lisa, la Vénus de Milo, la Victoire de Samothrace et le bâtiment lui-même.Dans notre recherche,nous avons constaté que les Brésiliens sont ravis de Mona Lisa et du musée lui-même.Nous pouvons clairement comprendre ce résultat: Mona Lisa est très présente dans l'imaginaire de l'art européen; le bâtiment,ses dimensions, ses ornements impressionnent surtout quant on se réfère aux modestes musées brésiliens. 
L'émotion liée à la visite d'un grand palais- fût-ce aujourd'hui désaffecté- est unique.Nous nous rendons compte que les Brésiliens, en particulier ceux qui ont récemment accedé à la classe moyenne et ont le privilège de voyager, se sentent très heureux d'être en mesure de visiter le Louvre.

Cependant, les Brésiliens seraient pleinement satisfaits si la signalétique et la signalisation du musée étaient plus claires, s'il y avait des professionnels multilingues et, surtout, des publications et des audioguides en portugais. Pour un public étranger qui ne connaît pas la langue française, ces dispositifs constitueraient un outil important pour l'amélioration de la compréhension du musée et de ses collections.

\section{References}

[1] CAILLET, Elisabeth LES POLITIQUES DE PUBLICS DANS LES MUSÉES Disponible en: http://documents.irevues.inist.fr/bitstream/handle/2042/14915/HERMES_1996_20_133.pdf;jsessionid=C2662233AB1 349696EAEC814F4F5E876? sequence=1 Accès: 7. nov 2014

[2] CARVALHO, Rosane Maria Rocha de. As transformações da relação museu e público sob a influência das tecnologias da informação. IN: MUSAS - Revista Brasileira de Museus e Museologia, n. 2. Rio de Janeiro, IPHAN, 2006. p. 127-139. ISSN: 1807-6149.

[3] CARVALHO, Rosane Maria Rocha de. A pesquisa de avaliação da exposição “A res publica brasileira” do Museu da República. Anais do XIV Encontro Nacional de Pesquisa em Ciência da Informação - XIV ENANCIB 2013.

Florianópolis, 2013. ISBN: 978-85-65044-06-

Disponible en: http://enancib2013.ufsc.br/index.php/enancib2013/XIVenancib/paper/viewFile/210/211 Accès: 18. nov 2014

[4] LEHALLE, Evelyne. Musées et visiteurs: un observatoire permanent des publics.1993. Disponible en http://doc.ocim.fr/LO/LO026/LO26\%283\%29-.PDF Accès: 5. nov 2014

[5] L'Observatoire des Publics du Musée du Louvre Disponible en : http://pt.slideshare.net/lapizmina/observatoire-despublics-du-louvre Accès: 7. nov 2014

[6] POULOT, Dominique. Patrimoine et musées: L'instituition de la culture. Hachette, 2001, Paris

[7] POULOT, Dominique. Une histoire des musées de France - XVIIIe-XXesiècles. La Decouverte, Paris, 2006

[8] SANTOS, Eloisa Pérez. Estudio de visitantes en museos: metodología y aplicaciones. Gijon (Asturias): EdicionesTrea, s. L., 2000

[9] TOBELEM, Jean-Michel. Le nouvel age des musées Les instituitions culturelles au défi de la géstion.Armand Colin, Paris, 2013. Disponible en: http://www.infraero.gov.br/index.php/br/estatistica-dos-aeroportos.html Accès: 21.oct 2014 